\title{
Altered serum levels of neprilysin in heart failure patients with reduced ejection fraction
}

\author{
Nemcekova $\mathrm{V}^{1,2}$, Malikova $\mathrm{E}^{1}$, Goncalvesova $\mathrm{E}^{3,4}$, Krenek $\mathrm{P}^{1}$, Klimas $\mathrm{J}^{1}$ \\ Department of Pharmacology and Toxicology, Faculty of Pharmacy, Comenius University in Bratislava, \\ Slovakia.jan.klimas@uniba.sk
}

\begin{abstract}
OBJECTIVE: In addition to the recent success of neprilysin inhibition in treatment of heart failure, elevated soluble neprilysin (SNEP) in circulation has been suggested to be a prognostic biomarker in heart failure with a reduced ejection fraction (HFrEF). However, the diagnostic performance of SNEP is nebulous and its levels in HFrEF have not been compared with controls. For the purpose of this study, we determined the role of sNEP levels as a biomarker in routine ambulatory care of HFrEF patients, when compared to the control subjects. METHODS: Ambulant patients with chronic $\operatorname{HFrEF}(n=18)$ were included. Apparently healthy volunteers - hospital physicians $(n=9)$ were included as the controls. Besides standard diagnostic tools (echocardiographic examination and laboratory biochemical diagnostic tests including NT-proBNP assessment), we analysed serum levels of neprilysin with a commercially available human soluble neprilysin ultrasensitive ELISA kit (Aviscera Bioscience, USA).

RESULTS: Concentrations of sNEP were significantly reduced in HFrEF patients (average \pm S.E.M. $=1038$ $\pm 464 \mathrm{pg} / \mathrm{ml}$ ) when compared to the controls $(1947 \pm 613 \mathrm{pg} / \mathrm{ml} ; \mathrm{p}<0.05)$. Two of eighteen HFrEF samples were below, while two of ten control samples were above the detection limit of the immunoassay. We documented a lack of significant correlation between sNEP and left ventricular ejection fraction (LVEF) and other echocardiographic features as well as NT-proBNP. However, sNEP significantly negatively correlated to serum natrium levels (Spearman $r=-0.6112, p<0.05)$ and to systolic blood pressure (Spearman $r=$ $-0.4746, p<0.05)$ in HFrEF.

CONCLUSION: Levels of SNEP were significantly reduced in HFrEF, when compared to the controls, with absent correlations to relevant HF-related features (e.g. LVEF). These findings might contribute to clarification of the diagnostic value of sNEP in HF (Tab. 2, Fig. 2, Ref. 30). Text in PDF www.elis.sk KEY WORDS: soluble neprilysin, heart failure, reduced ejection fraction, pharmacotherapy.
\end{abstract}

\begin{abstract}
Abbreviations: ACE - angiotensin-converting enzyme, Ang (1-7) - angiotensin (1-7), Ang II - angiotensin II, AT1R - angiotensin II type 1 receptor, $\mathrm{CON}$ - controls, HDL - high density lipoproteins, HF - heart failure, HFpEF - heart failure with preserved ejection fraction, HFrEF - heart failure with reduced ejection fraction, IVS $_{\mathrm{d}}-$ interventricular septum in diastole, LVEDD - left ventricular end-diastolic diameter, LVEF - left ventricular ejection fraction, NEP - neprilysin, NPs - natriuretic peptides, NS - not significant, NT-proBNP - N-terminal prohormone of brain natriuretic peptide, $\mathrm{PW}$ - posterior wall thickness, $\mathrm{sBP}$ - systolic blood pressure, sNEP - soluble neprilysin.
\end{abstract}

${ }^{1}$ Department of Pharmacology and Toxicology, Faculty of Pharmacy, Comenius University in Bratislava, Slovakia, ${ }^{2}$ St Michael's University Hospital, $2^{\text {nd }}$ Internal ambulance, Bratislava, Slovakia, ${ }^{3}$ Faculty of Medicine, Comenius University, Bratislava, Slovakia, and ${ }^{4}$ National Institute of Cardiovascular Diseases, Clinic of Cardiology, Department of Heart Failure, Bratislava, Slovakia

Address for correspondence: J. Klimas, Department of Pharmacology and Toxicology, Faculty of Pharmacy, Comenius University in Bratislava, Odbojarov 10, SK-832 32 Bratislava, Slovakia.

Phone: +421.2 .50117368$

Acknowledgments: This work was supported by a grant from the Slovak Research and Development Agency (APVV-15-0685).

\section{Introduction}

Neprilysin (NEP), a zinc-dependent cell-membrane-bound endopeptidase, is present in a variety of organs and cells including heart, kidneys and peripheral vasculature. As NEP can be released from the cell surface, it is present also in a soluble form (sNEP) in the circulation that retains catalytic activity (1). The enzyme cleaves a number of peptides (2), among the key in cardiovascular substrates to consider are the natriuretic peptides (NPs) and angiotensins, which are admittedly crucial in settings of heart failure (HF) (3). Concomitantly, NEP attenuates the antifibrotic, antiproliferative, cardiac, vascular, and diuretic properties of NPs by being responsible for removal of at least $50 \%$ of circulating NPs (4). Inhibition of NEP is accompanied by a reduction of NTpro B-type natriuretic peptide (NT-proBNP) levels (5), a surrogate marker of left ventricular wall stress which, however, is not cleaved by NEP (6).

Importantly, impressive benefits of combining NEP inhibition and angiotensin II type 1 receptor (AT1R) blockade have been demonstrated in the heart failure with a reduced ejection fraction (HFrEF) (7), what markedly turned the clinical attention to NEP. Though a slower clinical acceptation (8), real-world data also confirmed the effectiveness and safety of NEP inhibitor containing 
pharmacotherapy $(9,10)$, supporting that NEP plays an important role in the pathophysiology of HFrEF and accentuating NEP as an attractive biotarget in clinical research of HF. It is assumed that NEP levels and/or activity are elevated in settings of HF and, consequently, its inhibition slows the degradation of NPs, enhancing diuresis, natriuresis, myocardial relaxation and anti-remodelling, thus providing clinical benefit $(3,11)$.

NEP in a soluble form in blood has been increasingly investigated as a diagnostic tool and predictor of chronic HF outcome (12-14). Indeed, sNEP level was significantly associated with cardiovascular death or HF hospitalization in patients with HFrEF in a linear relationship (12), supporting the hypothesis that its inhibition may be beneficial in HFrEF. On the other hand, sNEP levels in $\mathrm{HF}$ with a preserved ejection fraction (HFpEF) were not found to be associated with attributes of $\mathrm{HF}$, including hospitalization and death (13). However, to understand its role as a biomarker, sNEP levels should be measured and compared to the control subjects (15). Interestingly, the study in HFpEF patients unveiled a rather lower circulating SNEP when compared to the controls (15) questioning its strength as a helpful biomarker.

Currently, blood concentration of NT-proBNP is one of the principal diagnostic tests in HF (3). Taking into account the independency of NT-proBNP and NEP, we hypothesized that serum neprilysin levels might provide an additional diagnostic utility in treated HFrEF. The aim of our small observational study was to determine the role of sNEP levels as a biomarker in a routine ambulatory care of HFrEF patients and its concentration in patients when compared to the apparently healthy control subjects.

\section{Methods}

\section{Study cohort}

From the medical database of ambulant patients managed at a private cardiology outpatient clinic containing 527 subjects, exclusively those with chronic HFrEF (LVEF with 25-35\% at the initial diagnostics), continuously followed using standard diagnostics tests (3), completing sampling and all examinations and pharmacologically treated for at least 36 months, who provided a written consent, were included $(n=18 ;$ males/females $=14 / 4)$. The study sample comprised mainly patients with HFrEF of ischemic aetiology ( $89 \%$ of subjects). The study was approved by local ethics committee (Ethics Committee of Faculty of Pharmacy, Comenius University in Bratislava). Apparently healthy volunteers, physicians from the hospital $(n=10$; males/females $=8 / 2)$ at the median age of 38 [interquartile range of 33-40] years provided control blood samples.

\section{Medical examination}

Patients were routinely examined at the cardiology outpatient clinic KARDIO-SANUS, s.r.o., in Bratislava, Slovak Republic. The investigation consisted of routine physical examination. In addition, patients underwent transthoracic echocardiographic measurement according to the current standards (16) and ambulatory blood pressure measurement. A New York Heart Association (NYHA) class was assigned to all the patients, who were in NYHA functional classes I (5\% of cases), II (53\%) and III (37\%) and IV (5\%).

\section{Blood samples}

Following examination, blood samples were routinely taken for blood count and standard laboratory diagnostic tests. The blood was taken between 7:00 and 8:00 a.m. after an overnight fasting. Biochemical examination, NT-proBNP levels and blood count were performed within a standard service provided by certified diagnostic laboratory (Medirex, a. s., Bratislava, Slovakia).

For the analyses of experimental biomarker, additional venous blood samples were collected to S-Monovette tubes containing Serum Gel with Clotting Activator (catalogue number: 04.1935). The samples were incubated at room temperature for $30 \mathrm{~min}$. Afterwards, they were centrifuged at $1500 \mathrm{G}$ for $15 \mathrm{~min}$ at $25{ }^{\circ} \mathrm{C}$. The serum samples were immediately stored at $-80{ }^{\circ} \mathrm{C}$ until further analysis.

\section{Soluble neprilysin assay}

Circulating sNEP was measured with a quantitative sandwich immunoassay (High-sensitivity Soluble Human Neprilysin ELISA kit, Aviscera Bioscience, Santa Clara, USA, code No: SK0072401, Lot: 20113789). Serum aliquots were diluted 1:4 in dilution buffer provided in the kit (DB03) before incubation. The ELISA was then performed according to the manufacturer's protocol. This assay displays $0 \%$ cross-reactivity with metallopeptidases, specifically, endothelin converting enzymes 1 and 2 . There was also no cross-reactivity with erythrocyte cell-surface antigen (KELL). Measurements were performed in duplicates.

Angiotensin II and Angiotensin (1-7) assays

Angiotensin II (Ang II) and angiotensin (1-7) (Ang (1-7)) were measured using a competitive ELISA method (Human Ang-II ELISA Kit, MyBioSource, San Diego, USA, Catalog No: MBS2506893, Lot: AK0017MAR30037; Human Ang (1-7) ELISA Kit, MyBioSource, San Diego, USA, Catalog No: MBS2515945). Serum aliquots were not diluted before the incubation. The ELISA was performed according to the manufacturer's protocol. The kits did not display any significant cross-reactivity between human Ang II/Ang (1-7) and all the analogues. Measurements were performed in duplicates.

\section{Statistical analysis}

Shapiro-Wilk test was used as normality test. For non-normally distributed data, we performed the Mann-Whitney test. Statistical correlation between two data groups was determined by Spearman's correlation coefficients. All data were handled by GraphPad Prism (GraphPad Software Inc., San Diego, CA, USA, version 6). The value of $p<0.05$ was considered statistically significant.

\section{Results}

Basic clinical features of tested sample are presented in the Table 1. Two of eighteen HFrEF samples were below, while two of ten control samples were above the detection limit of used sNEP 
Tab. 1. Basic clinical features of studied sample (number of subjects = 16; males $/$ females $=12 / 4$ ).

\begin{tabular}{|c|c|}
\hline & $\begin{array}{l}\text { Median } \\
\text { [25th-75th percentile] }\end{array}$ \\
\hline Age (years) & $74[62-82]$ \\
\hline Treatment duration (months) & $36[36-48]$ \\
\hline \multicolumn{2}{|l|}{ Echocardiography } \\
\hline LVEDD (mm) & $61[53-64]$ \\
\hline $\operatorname{IVSd}(\mathrm{mm})$ & $10[9-11]$ \\
\hline PWT (mm) & $10[10-110]$ \\
\hline Left atrial diameter $(\mathrm{mm})$ & $51[49-58]$ \\
\hline Right ventricular diameter (mm) & $35[30-36]$ \\
\hline Ascending aorta diameter $(\mathrm{mm})$ & $38[35-40]$ \\
\hline $\operatorname{LVEF}(\%)$ & $35[25-40]$ \\
\hline \multicolumn{2}{|l|}{ Blood pressures } \\
\hline Systolic blood pressure (mmHg) & 128 [124-144] \\
\hline Diastolic blood pressure (mmHg) & $80[69-85]$ \\
\hline Heart rate (beat per minute) & $79[66-86]$ \\
\hline \multicolumn{2}{|l|}{ Blood tests } \\
\hline NT-proBNP (ng/l) & 964 [477-2022] \\
\hline Ang II (pg/ml) & $1598[957-2488]$ \\
\hline Ang (1-7) (pg/ml) & $1241[716-1506]$ \\
\hline Glucose $(\mathrm{mmol} / \mathrm{l})$ & $5.64[5.23-7.08]$ \\
\hline Creatinine $(\mu \mathrm{mol} / \mathrm{l})$ & $104[87-128]$ \\
\hline Uric acid $(\mathrm{mmol} / \mathrm{l})$ & $0.35[0.30-0.43]$ \\
\hline Total cholesterol $(\mathrm{mmol} / \mathrm{l})$ & $3.63[3.09-4.93]$ \\
\hline $\mathrm{HDL}(\mathrm{mmol} / \mathrm{l})$ & $1.13[0.99-1.39]$ \\
\hline Triglycerides (mmol/l) & $1.32[0.84-1.76]$ \\
\hline Sodium $(\mathrm{mmol} / \mathrm{l})$ & $140[139-143]$ \\
\hline Potassium $(\mathrm{mmol} / \mathrm{l})$ & $4.60[4.09-4.85]$ \\
\hline White blood cell count (109/1) & $7.90[7.33-8.45]$ \\
\hline Platelet count $(109 / 1)$ & $213[185-245]$ \\
\hline Red blood cell count $(1012 / 1)$ & $4.13[4.08-4.72]$ \\
\hline Haematocrit (\%) & $40[40-44]$ \\
\hline Haemoglobin $(g / 1)$ & $136[131-148]$ \\
\hline Mean cell haemoglobin (pg) & $32[31-33]$ \\
\hline Mean cell volume (fl) & $97[91-99]$ \\
\hline
\end{tabular}

assay. These subjects were not included in further analyses. Thus, the analysed sample consisted of $16 \mathrm{HFrEF}$ patients (males/females $=12 / 4)$ and 8 controls (6/2). Among them, four patients and four volunteers reported smoking history.

At the clinical examination time, the pharmacotherapy of patients (table 2) consisted mostly of beta-blockers, ACE inhibitors

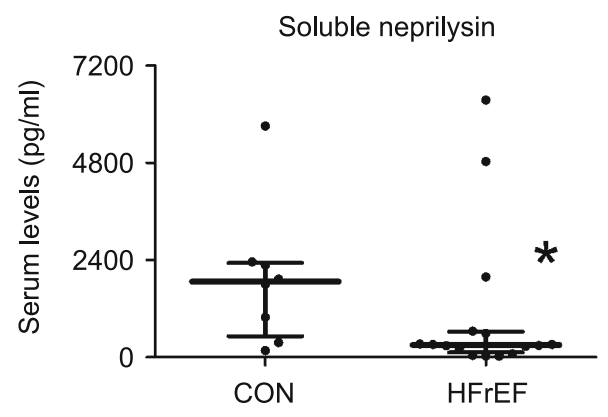

Fig. 1. Serum levels of soluble neprilysin in healthy volunteers (CON, $n$ $=8$ ) and heart failure patients with reduced ejection fraction (HFrEF, $\mathbf{n}=16$ ). Figure depicts particular values, lines represent median with interquartile range. ${ }^{*} \mathbf{p}<0.05$.
Tab. 2. Pharmacological treatment at the time of physical examination and determination of sNEP.

\begin{tabular}{lc}
\hline & $\begin{array}{c}\text { Number of patients taking } \\
\text { the medication }\end{array}$ \\
\hline Beta-blocker & 12 \\
ACE inhibitor or AT1R antagonist & 13 \\
Aldosterone antagonist & 13 \\
Diuretic & 13 \\
Calcium channel blocker & 3 \\
Ivabradine & 1 \\
Digoxin & 3 \\
Nitrate & 6 \\
\hline
\end{tabular}

or AT1 receptor antagonists, aldosterone antagonists (eplerenone or spironolactone) and furosemide (the only taken diuretic).

We observed significantly reduced levels of circulating neprilysin, when compared to healthy volunteers (by $47 \%$ of arithmetic means, $\mathrm{p}<0.05$; medians with interquartile ranges are depicted in Figure 1). We observed a lack of any significant correlation between serum neprilysin levels and HFrEF most relevant features (i.e. NYHA class, LVEF and NT-proBNP) (Fig. 2), however, a significantly negative relation of SNEP to sodium concentrations and also to systolic blood pressures appeared ( $\mathrm{p}<0.05$ for both) (Fig. 2).

\section{Discussion}

Recently, NEP became a meaningful biotarget in cardiology (7), with a potential to be a prognostic biomarker $(12,17)$. However, its utility as a diagnostic tool is still uncertain $(13,15,17,18)$. In this study, we documented lower sNEP levels in treated HFrEF patients, when compared to the apparently healthy controls. This finding is strengthened by the fact that two HFrEF patients from the initial sample had sNEP values below the detection limit of used immunoassay, while two of controls dropped out from the statistical analyses due to SNEP concentrations above the reliable detection range of the assay used. In addition, sNEP levels also failed to correlate with the main features of HFrEF, such as: NTproBNP and LVEF questioning whether circulating sNEP concentration could be a reliable biomarker in HFrEF.

Reduced sNEP levels in HFrEF, when compared to the controls might be perceived as a consequence of its reduced release from the failing myocardium. Although the predominant source of sNEP in patients with HF remains unclear, Arrigo et al. indicated that the heart constitutes the major source of sNEP in patients with HFrEF (19). In contrast, others reported only a weak cardiac relation to SNEP levels (20) what raises the alternative, inter alia, that sNEP levels were influenced by acute substantial changes in systemic haemodynamics rather than by cardiac status. Consistent with this idea, NEP is widely expressed in various tissues other than the heart and the reduction of sNEP might be a consequence of changed release at other sites. One of the promising explanation might involve the lungs, as smoking history was shown to be a substantial suppressor of NEP expression in lung tissue (21), in accordance with findings of lower circulating NEP 

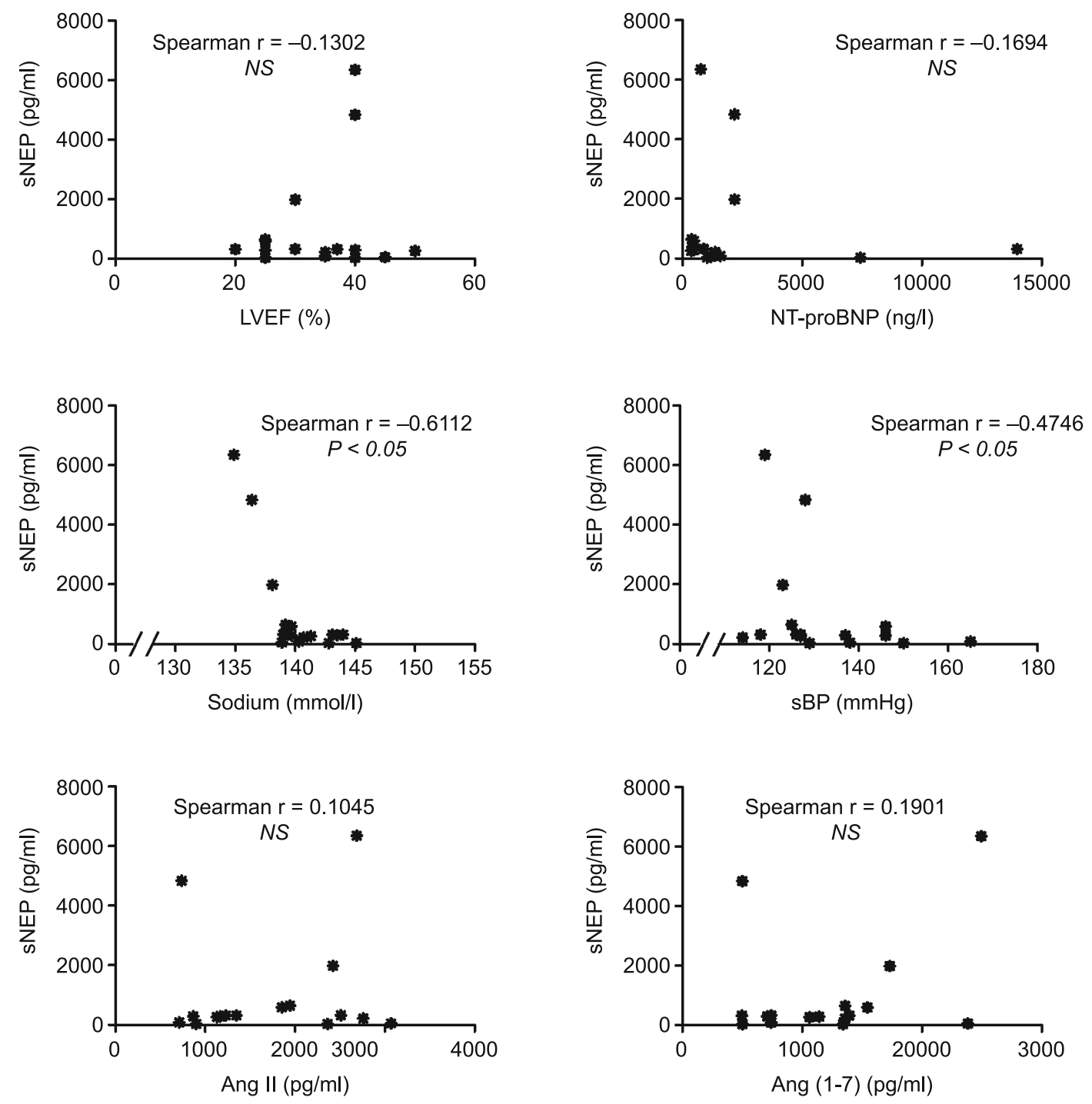

Fig. 2. Relationship between circulating soluble neprilysin levels and their potential clinical and pathophysiological correlates in HFrEF patients $(n=16)$.

levels in smokers $(13,15)$. An alternative explanation of reduced sNEP is the modulation of sNEP levels by comorbidities. In renal complications, however, authors documented a link of sNEP activity, but not of SNEP plasma concentration, to HF related features $(14,22)$. Also, sNEP levels are less affected by obesity, age, sex, or functional/clinical status $(12,17,18,23,24)$, what should underline some potential advantages over standard biomarkers for HF such as natriuretic peptides.

In general, it is difficult to estimate the value of the observed reduction in SNEP as previous studies on the positive association of sNEP with cardiovascular mortality and morbidity in HFrEF patients did not provide control data $(12,17)$. Similar to our findings, moderately lower values of sNEP were documented in HFpEF, when compared to the controls (15). Interestingly, reduced sNEP levels were associated with diastolic dysfunction, dyslipidaemia, and hypertension also in general population (18). Still, it is challenging to estimate the pathophysiological consequence of reduced SNEP because of the complexity of the NEP-NPs pathway. NEP is very nonspecific in terms of substrate selection (note that NEP activity affects the degradation of more than 50 vasopeptides) and breaks down both beneficial peptides in addition to harmful substrates (2), e.g. natriuretic peptides have vasodilatory effects, while angiotensin II is a vasoconstrictor. Therefore, the pathophysiological role and levels of sNEP may differ, depending on the complex interplay of these various substrates in the pathophysiology of the HF syndrome. The observed negative correlations of sNEP to sBP and $\mathrm{Na}^{+}$likely mirror this complexity.

An association between sNEP levels and adverse outcomes in heart failure patients has been demonstrated $(12,17)$. However, a lack of association between sNEP and cardiac structure, or function 
is seen in diverse HF phenotypes and also in general population $(13,15,17,18,25)$. Our findings in HFrEF patients also support the view that sNEP levels are not directly related to cardiac dysfunction. Though we have not measured the activity of SNEP in this study, we admit its importance in HFrEF. The levels of sNEP have been also introduced as a reliable surrogate for neprilysin activity (26). On the contrary, circulating sNEP activity, but not concentration has been suggested to provide an incremental prognostic information (14).

In general, the observed lack of direct relationship between SNEP and NT-proBNP levels may be anticipated and is in line with the findings of others $(15,18,24)$. Firstly, neprilysin is neither involved in NT-proBNP cleavage, nor its biosynthesis pathway (6). Secondly, biologically active B-type natriuretic peptide directly inhibits sNEP activity, but not sNEP concentration in HF patients (27). Thirdly, complications, such as impaired renal function, may differentially influence the levels of both biomarkers (24). Consequently, the use of sNEP, simultaneously to NT-proBNP as a gold standard biomarker in HF and as a default comparator, when investigating novel biomarkers and therapies (28-30), may be of diagnostic advantage providing an additional valuable prognostic information for identifying diverse HF patient subpopulations. However, less sNEP in HFrEF sheds a new light on this hypothesis.

\section{Limitations}

There are certain limitations in the present study. Firstly, this is an observational single-centre study realized in a small sample of patients. These conditions may markedly influence the applicability of our results to larger population. Secondly, there are serious analytical issues with respect to SNEP measurement, as i) there is little information about the stability of sNEP, ii) requirements for preanalytical management of samples is not well defined, and iii) data comparisons among the different commercially available immunoassays have revealed a lack of reproducibility (1). This must be resolved before its implementation in a routine clinical practice. Thirdly, our study sample comprised mainly patients with HFrEF of ischemic aetiology. The possible extrapolation to diverse HF subpopulations and/or to the entire spectrum of patients with HF is uncertain. Fourthly, the lack of repetitive blood sampling precludes the complex view on the kinetics of sNEP during the disease development and on the effect of pharmacotherapy.

\section{Conclusion}

Conclusively, sNEP levels were significantly reduced in HFrEF patients, when compared to healthy individuals and their correlation to HF-related features (e.g. LVEF) is rather absent. These data shed new light on our simplistic assumptions of sNEP as a potential biomarker in HFrEF being in contrast to its recent importance as a target in the treatment of HF. Despite the limited sample size in this study, the observed finding of sNEP deficiency might contribute to clarification of the value of sNEP in HF diagnostics.

\section{References}

1. Bayes-Genis A, Barallat J, Richards AM. A Test in Context: Neprilysin: Function, Inhibition, and Biomarker. J Am Coll Cardiol 2016; 68 (6): 639-653.

2. Pankow $\mathrm{K}$ et al. Structural substrate conditions required for neutral endopeptidase-mediated natriuretic Peptide degradation. J Mol Biol 2009; 393 (2): 496-503.

3. Ponikowski P et al. 2016 ESC Guidelines for the diagnosis and treatment of acute and chronic heart failure: The Task Force for the diagnosis and treatment of acute and chronic heart failure of the European Society of Cardiology (ESC) Developed with the special contribution of the Heart Failure Association (HFA) of the ESC. Eur Heart J 2016; 37 (27): 2129-2200.

4. Charles CJ et al. Clearance receptors and endopeptidase 24.11: equal role in natriuretic peptide metabolism in conscious sheep. Am J Physiol 1996; 271 (2 Pt 2): R373-R380.

5. Solomon SD et al. The angiotensin receptor neprilysin inhibitor LCZ696 in heart failure with preserved ejection fraction: a phase 2 double-blind randomised controlled trial. Lancet 2012; 380 (9851): 1387-1395.

6. Semenov AG, Katrukha AG. Different Susceptibility of B-Type Natriuretic Peptide (BNP) and BNP Precursor (proBNP) to Cleavage by Neprilysin: The N-Terminal Part Does Matter. Clin Chem 2016; 62 (4): 617-622.

7. McMurray JJ et al. Angiotensin-neprilysin inhibition versus enalapril in heart failure. N Engl J Med 2014; 371 (11): 993-1004.

8. Farmakis D et al. Practical considerations on the introduction of sacu$\mathrm{bitril} / \mathrm{valsartan}$ in clinical practice: Current evidence and early experience. Int J Cardiol 2016; 223: 781-784.

9. Martens $\mathbf{P}$ et al. Insights into implementation of sacubitril/valsartan into clinical practice. ESC Heart Fail 2018; 5 (3): 275-283.

10. Chang $\mathrm{HY}$ et al. Sacubitril/valsartan in heart failure with reduced ejection fraction patients: Real world experience on advanced chronic kidney disease, hypotension, and dose escalation. J Cardiol 2019; 74 (4): 372-380.

11. Mangiafico $S$ et al. Neutral endopeptidase inhibition and the natriuretic peptide system: an evolving strategy in cardiovascular therapeutics. Eur Heart J 2013; 34 (12): 886-893.

12. Bayés-Genís A et al. Soluble neprilysin is predictive of cardiovascular death and heart failure hospitalization in heart failure patients. J Am Coll Cardiol 2015; 65 (7): 657-665.

13. Goliasch G et al. Soluble neprilysin does not correlate with outcome in heart failure with preserved ejection fraction. Eur J Heart Fail 2016; 18 (1): 89-93.

14. Feygina EE, Katrukha AG, Semenov AG. Neutral Endopeptidase (Neprilysin) in Therapy and Diagnostics: Yin and Yang. Biochemistry (Mosc) 2019; 84 (11): 1346-1358.

15. Lyle MA et al. Circulating Neprilysin in Patients With Heart Failure and Preserved Ejection Fraction. JACC Heart Fail 2020; 8 (1): 70-80.

16. Galderisi $M$ et al. Intra- and interobserver reproducibility of Dopplerassessed indexes of left ventricular diastolic function in a populationbased study (the Framingham Heart Study). Am J Cardiol 1992; 70 (15): 1341-1346.

17. Núñez $\mathbf{J}$ et al. Serum neprilysin and recurrent hospitalizations after acute heart failure. Int J Cardiol 2016; 220: 742-744. 
18. Reddy YNV et al. Soluble Neprilysin in the General Population: Clinical Determinants and Its Relationship to Cardiovascular Disease. J Am Heart Assoc 2019; 8 (15): e012943.

19. Arrigo $\mathbf{M}$ et al. The heart regulates the endocrine response to heart failure: cardiac contribution to circulating neprilysin. Eur Heart J 2018; 39 (20): 1794-1798.

20. Takahama H, Minamino N, Izumi C. Plasma soluble neprilysin levels are unchanged during recovery after decompensation of heart failure: a matter of the magnitude of the changes in systemic haemodynamics? Eur Heart J 2018; 39 (37): 3472-3473.

21. Wick MJ et al. Decreased neprilysin and pulmonary vascular remodeling in chronic obstructive pulmonary disease. Am J Respir Crit Care Med 2011; 183 (3): 330-340.

22. Emrich IE et al. Do plasma neprilysin activity and plasma neprilysin concentration predict cardiac events in chronic kidney disease patients? Nephrol Dial Transplant 2019; 34 (1): 100-108.

23. Bayés-Genís A et al. Prognostic Value and Kinetics of Soluble Neprilysin in Acute Heart Failure: A Pilot Study. JACC Heart Fail 2015; 3 (8): 641-644.
24. Bayes-Genis A et al. Multimarker Strategy for Heart Failure Prognostication. Value of Neurohormonal Biomarkers: Neprilysin vs NT-proBNP. Rev Esp Cardiol (Engl Ed) 2015; 68 (12): 1075-1084.

25. Moliner P et al. Bio-profiling and bio-prognostication of chronic heart failure with mid-range ejection fraction. Int J Cardiol 2018; 257: 188-192.

26. Bayes-Genis A et al. Soluble neprilysin retains catalytic activity in heart failure. J Heart Lung Transplant 2016; 35 (5): 684-685.

27. Vodovar $\mathbf{N}$ et al. Elevated Plasma B-Type Natriuretic Peptide Concentrations Directly Inhibit Circulating Neprilysin Activity in Heart Failure. JACC Heart Fail 2015; 3 (8): 629-636.

28. McKie PM, Burnett JC Jr. NT-proBNP: The Gold Standard Biomarker in Heart Failure. J Am Coll Cardiol 2016; 68 (22): 2437-2439.

29. Demir $S$ et al. The novel diagnostic marker in low-LVEF heart failure patients. Bratisl Lek Listy 2018; 119 (7): 421-424.

30. Cunningham JW et al. Effects of Sacubitril/Valsartan on N-Terminal Pro-B-Type Natriuretic Peptide in Heart Failure with Preserved Ejection Fraction. JACC Heart Fail 2020; 8(5): 372-381.

Received June 1, 2020. Accepted October 26, 2020. 